\title{
Application of Sequence Comparison Techniques To Multisensor Data Fusion and Target Recognition
}

\author{
E. W. Libby ${ }^{t}$ and P. S. Maybeck \\ Air Force Institute of Technology \\ Wright-Patterson Air Force Base, OH 45433-7765
}

\begin{abstract}
A new class of techniques for multisensor fusion and target recognition is proposed using sequence comparison by dynamic programming and multiple model estimation. The objective is to fuse information on the kinematic state and "nonkinematic" signature of unclassified targets, assessing the joint likelihood of all observed events for recognition. Relationships are shown to previous efforts in pattern recognition and state estimation. This research applies "classical" speech processing-related and other sequence comparison methods to moving target recognition, extends the efforts of previous researchers through improved fusion with kinematic information, relates the proposed techniques to Bayesian theory, and applies parameter identification methods to target recognition for improved understanding of the subject in general. The proposed techniques are evaluated and compared to existing approaches using the method of generalised ambiguity functions, which leads to a form of Cramer-Rao lower bound for target recognition.
\end{abstract}

\section{Introduction}

Several authors in the multisensor fusion field have proposed using kinematic and nonkinematic information for "observation-to-track" assignment or target recognition [3:177-178] [7]. In [2:297-320], Mitsel proposed the use of linear estimation techniquea with target state vectora containing both kinematic and nonkinematic states, but his development treated these states as independent. In contrast, the motion fusion research diecused here seeks to exploit the fact that nonkinematic or "signature" states, which are in general nearly direct function of target-to-neneor apect angle, are tightly coupled to target kinematica, in particular for turning targets. We are also interested in state-measurement relationshipa that are not well modelled with linear estimator.

The key step in this research was to consider the relationship between true and estimated kinematica and signatures for a track-propelled vehicle (e.g., a tank) in a turn. Unlike wheeled vehicles, tracked vebicles do not turn with a constant or even continuous radius of curvature, and in fact their motion can be described as nearly piecewise linear [13]. Consider the process of tracking a tank in a planar turn. Our sampled-data sensor providea a sequence of signature vectors, as measured at discrete times over the observation period. Concurrently, from conventional range/angle tracking and kinematic state estimation alone, for any feacible target class, we can hypotheaise a sequence of anticipsted signature voctors. Comparing the observed sequence to the kinematically-estimated sequence for the correct target, we see that their differences can be deacribed fundamentally in terms of expansions and contractions of one sequence relative to the other. Can we compare these two nequences such that their origin target clases are seen to be identical, despite expansions and contractions?

This is a well-known problem in speech recognition, where the technique of "Dynamic Time Warpins" (DTW) using dynamic programming (DP) $[6,12]$ for sequence comparison evolved during the 1970 's $[27,28,30]$. The objective in that field was to quantify the eimilarity of two apolen words, despite pronunciation differences. For DTW comparison, words are represented by vector sequences of finite but varied length, extracted from apeech by any of several algorithm [27]. As in the "turning tank" example above, the differences between sequences describing the same word from different sources may be claceified as expansions, contractions, and perhaps insertions and deletions [30].

As we began to develop DP-based sequence comparieon for moving target recognition, the Target Recognition Technology Branch (WL/AARA) of the USAF's Wright Laboratory requeated that the technique be applied to aircraft recognition using high-range resolution radar (HRRR). An exhaubtive literature search was conducted, covering the areas of multisensor furion, dynamic programming, and pattern recognition. The text by Sankoff and Kruskal [30] demonstrated the wide utility of what we will call "clanoical"

tPlease address all correspondence to the second author.
DP-based sequence comparison techniques, including DTW. Efforts by Barniv [2:85-154] illuminated a DP algorithm originally developed by Larson and Peschon [18] for state estimation. This "Larwon and Peschon (L\&P) algorithm" and classical DP sequence comparison will be shown below to be intimately related, and both were applied in our research.

Even a brief literature review shows that DP and DTW techniques are not new in pattern recognition $[11,16]$. However, the authors found only two sources which appeared to any extent to share our methods and objectives. The first was a 1978 article by Le Chevalier et al. [19], who proposed a real-time "Byntactic" radar target recognition system using the "well-known shortest path algorithm" (i.e., dynamic programming), approximate target aspect angle from kinematics, and knowledge of target kinematic bounds to assign sequences of signatures to the correct target class.

The second source was a classified paper [25] by Mieras et al. of Raytheon, whose approach appears to be fundamentally identical to Le Chevalier's although independently conceived. It will be shown in this paper that the $L e$ Chevalier and Mieras approaches can be posed as "suboptimal" applications of the L\&P algorithm, and it appears that published applications of this algorithm provided the original inspiration for Mieras et al.

\section{Classical Pattern / Target Recognition}

Classical pattern recognition [14] generally follows two basic approsches "decision theoretic" (i.e., parametric or statistical, and nonparametric or nearest neighbor concepts) and "syntactic". Syntactic methods consider the order of presentation of "features" (observed quantities) by an unclasaified object relative to those of known clnseen, while decision theoretic classifier simply consider the closeness (in some metric) of the unclassified object's features to those of known classes, irreapective of order. Speech recognition approaches are almost invariably syntactic, and syntactic pattern recognition theory derives largely from the study of languages. Most tactical target recognition approaches today are decision theoretic: a few are syntactic, including those using DTW techniques (e.g., [16]).

Le Chevalier et al. appears to have been the first to apply a syntactic, grammatical approach to recognition of moving targets. This approach independently conceived and extended by Mieras ot al. and the authors, deserves much wider attention and application than it appears to have received in the tactical target recognition world.

A typical decision theoretic classifier for tactical targets consists of a library of signatures recorded for known targets at discrete aspect angles. In the absence of a priori information as to the aspect angle of an unclassified target, a target is considered to belong to the known class for which the closest match is found between measured and library signatures. To reduce library search requirements and the posibility of incorrect matches, decision theoretic associations are often limited to aspect angle "windows" of given solid angle extent. Multiple signature observations can be used with classical techniques (e.g., Bayes' Rule, voting rules, etc.) to provide better estimates of class membership for unknown targets.

For any set of signature realisations, this matching process inherently defines a "maximum likelihood" (ML) estimate of target aspect angle (a pose estimate) over time for each library class. This ML aspect angle history for each candidate class contains much useful information, but evidently has never been used explicitly for classificstion purposes, although some have so suggested [4]. Claseical decision theoretic classifiers are ill-suited to use this information. It will be seen that the Le Chevalier and Mieras approaches use this information implicitly, and our research has explored other means of doing $s$.

\section{Sequence Matching By DP}

The Dynamic Time Warping (DTW) and Larson and Peschon (L\&P) algorithms form the core of the moving target recognition approaches principally 
investigated by the authors to date. This section discusses them, their similarities and their differences.

\subsection{Classical Sequence Comparison / DTW}

In classical sequence comparison, generally all that we require of the sequence elements is that some distance metric exists by which one element can be compared to another. Each element will represent a discrete, sampled data representation from some feature space. The features generally represent observable quantities due to a physical (i.e., classically continuous) process or trajectory in some state space, where the true location in the state space at any time is unknown - the distinction between the state space of this trajectory and the feature space of the observables is an important one, and not always clear in the literature. Pausing to note that classical DP sequence comparison is a large class of algorithms including DTW, in the interest of space hereafter we will use the term DTW to refer to classical sequence comparison in general.

"Warping" or sequence comparison is the process of making association between individual elements in the two sequences, computing the cost of each association according to the distance (measure of dissimilarity) between the element in one sequence and the element in the other, and finding the set of associations that gives the minimum total cost or distance. Associations are made subject to "continuity constraints," that limit, for example, the number of associations that can be made from one element of one sequence to elements of the other sequence, the number of elements that can be skipped, and so on. Continuity constraints prevent undesirable low cost associations between two sequences that really have significant differences.

This process can be posed as finding the minimum cost path through a space of associations, subject to transition constraints, and forward dynamic programming [12:10-11] provides a natural approach to determine this path. For the simplest form of "local" continuity constraint, the forward dynamic programming cost computation at each step can be written as:

$$
D\left(C_{k}\right)=d\left[c_{k}\right]+\operatorname{MIN}\left[D\left(C_{k-1}\right)\right]
$$

where:

$c_{k}=\left[a_{j}, b_{l}\right]$ is the $k$-th element in a sequence of associations of elements from sequence $A$ ( $m$ elements in number) with elements of sequence $B$ ( $n$ elements), this association being between element $a_{j}$ and element $b_{l}$

$C_{k}=\left\{c_{1}, c_{2}, c_{3}, \ldots, c_{k}\right\}$, the minimum cost sequence of associations leading to and including association $c_{k}$

$d\left[c_{k}\right]=$ the cost or distance of association $c_{k}$, i.e., the distance in some metric between element $a_{j}$ and element $b_{1}$

$D\left(C_{k}\right)=$ the total cost of reaching and accomplishing association $c_{k}$ by the minimum cost sequence of allowable association

Feature space representations, distance metrics, and path constraints have been the subject of much experiment, without identification of one particular "best" approach $[27: 297-303]$ [30:125-161]. A particular issue faced by DTW researchers is that of warping path length compensation - inherently, the algorithm above is biased toward solutions with a minimum number of associations. Where a substantial difference exists between $m$ and $n$ or where $m$ and $n$ are equal but the optimal association "path" is highly nonlinear, classical DTW may be hard pressed to choose that association.

\subsection{The Larson and Peschon (L\&P) Algorithm.}

Larson and Peschon proposed an algorithm [18] for estimating the sequence of $n$ states or locations in some space with maximum a posteriori or MAP probability of producing an observed sequence of $n$ measurements, conditioned on a priori information about transitions in the state space. They did not motivate their work as a tool for target recognition working on an aspect angle space, but we will apply it in this fashion.

Given a sequence of measurements $\mathbf{z}_{k}=\left\{\mathbf{z}_{1}, \mathbf{s}_{2}, \ldots, \mathbf{s}_{k}\right\}$, Larson and Peschon desired to find the sequence of states $\hat{\mathbf{X}}_{k / k}=\left\{\hat{\mathbf{x}}_{0 / k}, \hat{\boldsymbol{x}}_{1 / k}, \hat{\boldsymbol{x}}_{2 / k}, \ldots\right.$ $\left.\hat{\mathbf{x}}_{k / k}\right\}$ that maximired the conditional probability density function:

$$
\begin{aligned}
& p\left(\hat{\mathbf{X}}_{\boldsymbol{k} / \boldsymbol{k}} \mid \mathbf{Z}_{\boldsymbol{k}}\right)= \\
& \operatorname{MAX}\left[p\left(x_{0}, x_{1}, \ldots, x_{k} \mid s_{1}, \ldots, s_{k}\right)\right]=\operatorname{MAX}\left[p\left(\mathbf{X}_{k} \mid z_{k}\right)\right]
\end{aligned}
$$

where the term "MAX" refers to the operation of finding the maximum value of the indicated term, over all values of $X_{k}$, representing the sequence of states $\left\{x_{0}, x_{1}, x_{2}, \ldots, x_{k}\right\}$. Note, as do Larson and Peschon, that the intent here is to estimate the entire sequence up to the present, rather than simply the present state $\mathbf{x}_{\boldsymbol{k}}$.

Next, Larson and Peschon were willing to assume independence of measurements $z_{j}$ from states $x_{l}$ and measurements $z_{l}$ for $t_{j} \neq t_{l}$, implying, for example, that the time interval required to take data for one measurement is less than and synchronised with the loiter time in any one state, and that the measurement instrument is independent from event to event. With this assumption, then, they used Bayes' Rule to break the maximisation process into stages, making it suitable for solution by dynamic programming using the following equations (use of which will be discussed below);

$$
\begin{aligned}
p\left(\hat{\mathbf{X}}_{k / k} \mid \mathbf{Z}_{k}\right) & =\left\{\begin{array}{cc}
\operatorname{MAX} & \operatorname{MAX}\left[p\left(\mathbf{X}_{k} \mid \mathbf{Z}_{k}\right)\right] \\
\mathbf{x}_{k} & \mathbf{X}_{k-1}
\end{array}\right\} \\
& =\left[\begin{array}{c}
\operatorname{MAX} I\left(x_{k}, k\right) \\
\boldsymbol{x}_{k}
\end{array}\right]
\end{aligned}
$$

which shows the final step in the process, a maximization of $I\left(x_{k}, k\right)$ over all possible final states $x_{k}$, where:

$$
I\left(\mathbf{x}_{k}, k\right)=\underset{\mathbf{X}_{k-1}}{\operatorname{MAX}}\left[p\left(\mathbf{X}_{k} \mid \mathbf{z}_{k}\right)\right]
$$

Then, stepping theoretically to a hypothetical $k+1-$ st step:

$$
I\left(\mathbf{x}_{k+1}, k+1\right)=\underset{\mathbf{X}_{k}}{\operatorname{MAX}}\left[\frac{p\left(s_{k+1} \mid \mathbf{x}_{k+1}\right) p\left(\mathbf{x}_{k+1} \mid \mathbf{x}_{k}\right)}{p\left(\mathbf{s}_{k+1} \mid \mathbf{Z}_{k}\right)} p\left(\mathbf{X}_{k} \mid \mathbf{Z}_{k}\right)\right]
$$

or, equivalently, in the recursive form which is the heart of the algorithm:

$$
I\left(x_{k+1}, k+1\right)=\underset{x_{k}}{\operatorname{MAX}}\left[\frac{p\left(z_{k+1} \mid x_{k+1}\right) p\left(x_{k+1} \mid x_{k}\right)}{p\left(z_{k+1} \mid \mathbf{Z}_{k}\right)} I\left(x_{k}, k\right)\right]
$$

The above equations are used in an recursive forward dynamic program ming procedure which works as follows (from [18] with elaboration):

(1) Quantise the state space $[x, t]$ to obtain a grid consistent with the accuracy requirements of the problem.

(2) Initialise the (forward DP) iterative procedure by defining $I\left(x_{0}, 0\right)=$ $p\left(x_{0}\right)$, the a priori density for each possible discrete $x$ at time $t_{0}$.

(3) For each quantised state $x_{1}$ (i.e., each possible discrete $x$ at time $t_{1}$ ) calculate $I\left(x_{1}, 1\right)$ from $s_{1}$ and Eq. (6), with appropriate subscript changes for stage 1, rather than $k+1$.

(4) Write $\dot{x}_{0}\left(x_{1}, 1\right)$ as the value of $x_{0}$ for which Eq. (6) is maximized in the previous calculation (establishing "pointers" which will be retraced to find the optimum state sequence)

(5) Repeat steps (3) and (4) at each sampling instant until the $k$-th instant is reached. Each repetition is one stage. This is the iterative forward dynamic programming procedure, moving forward through successive stages.

(6) Determine the modal trajectory $\hat{\mathbf{x}}_{k / k}$ by first using Eq. (3) to find $\hat{x}_{k / k}$ (i.e., the state with higheat probability of being the terminus of the true state sequence) and then iteratively retracing the pointers set up in $\operatorname{step}(\mathrm{s})$ (4), to find the optimal state sequence, i.e., $\hat{x}_{i / k}=\hat{x}_{i}\left(\hat{x}_{i+1 / k}, i+1\right)$.

Since the factor $p\left(\mathbf{s}_{k+1} \mid \mathbf{z}_{k}\right)$ is the same for all maximisations made at any time $t_{k}$, the actual maximisation at any stage need not be done over the term shown in braces in Eq. (6), but rather only over the expression defined by computing this term without its denominator, denoted $I^{*}\left(x_{k+1}, k+1\right)$.

\subsection{Relating DTW and the L\&P Algorithm.}

Both DTW and the L\&P algorithm are DP sequence comparison techniques. The fundamental difference between them is that DTW does not consider state transitions that occur off a single "one-dimensional" path in state space. In the usual DTW case, we have little knowledge of the underlying state space - only examples of the feature sequences produced by typical state trajectories. Observations from one state trajectory are simply compared to observations from another trajectory, and "warped" to allow for an optimal match. DTW generally attempts to associate an element of one sequence with more than one element of the other sequence, leading toward a bias for solutions that minimize the total number of associations.

On the other hand, the L\&P algorithm can use information known a priori, or aside from the feature observations, about the likelihood of transitions in the state space. This allows the L\&P algorithm to "investigate" more than one state trajectory. The L\&P algorithm does not attempt to match more than one state space point with a given element in the feature sequence, and thus has no "arithmetic" bias toward short paths in the state space.

The drawback to the L\&P algorithm is its "maximum likelibood" (ML) nature, in the sense that, given a set of $m$ observations, it finds the set of $m$ discrete states most likely to have generated the observations, subject to a 
priori constraints : $p\left(x_{0}\right)$ and $p\left(x_{k+1} \mid x_{k}\right)$. It may be, however, that a state space region exists which has a higher overall probability of producing the given observations, when all possible trajectories over time through that regrion are considered. By comparison with a region choeen by the L\&P algorithm, this "better" region might have many points which are rather likely to have originated the given observations, while the "L\&P" region has a few well-positioned points which are very likely origin, but many that are quite unlikely. The use of DTW in such a case, forcing each point along a likely state trajectory to associate with an observation, could select the "better" region instead of that selected by the L\&P algorithm.

Unfortunately, while the L\&P algorithm can uae the (reiative) computational economy of DP to find the ML sequence of atates in a state apace of arbitrary dimention, the ntate space region with higheat probability of generating the obeerved features can in general be found only by exhauntive search. A set of nominal or a priori likely trajectories through the atate space would provide a starting point for such a search with DTW methods. The next section develope these ideas formally.

\section{Moving Target Recognition: Theory}

Using the classical Bayenian approsch, we really deaire to produce a pattern

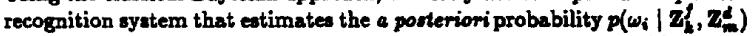
that we are actually observing a target of clase wi given a set of $k$ feature

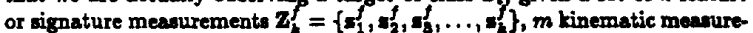
ments $Z_{m}^{d}=\left\{x_{1}^{d}, x_{2}^{d}, x_{3}^{d}, \ldots, x_{m}^{d}\right\}$ (note use of superacript $\alpha, a s$ in "dynamic", since $k$ is a counting index in the L\&P form), and a priori target claes probabilities $p\left(\omega_{i}\right)$ for each of $J$ known target classes.

This ideal, but in practice unobtainable, syatem would consiat of $J$ functions, one for each target clasa, having a domain of the space of all measurements over time and a range of the interval on the real line from sero to one, with the sum of the $J$ function values equal to one (or leas than one, if we wish to allow for unknown clases). Following Rao [29:353], however, in the abmence of $p\left(w_{i} \mid \mathbf{z}_{k}^{f}, \mathbf{z}_{m}^{d}\right)$ (or equivalently, the joint probubility deneity of target clas and measurements), we are content to find a vet of "generalised likelihood functiona such that the maximum value for each function is attained for the correct combination of target class and meanrements. Note that the target aignature libraries and anpect angle "windown" used in classical ATR are likelihood functions.

What can we gain by considering the kinematics of the unknown target? Consider an abstract space $O$ of all possible target models and aspect angles over time as part of the domain of a matching function, $C$. A clacaical ATR likelihood function for a given target class $\omega_{i}$ is defined by restricting the domain of $\mathcal{L}$ to produce an $\mathcal{L}_{i}$ with domain $S_{i} \subseteq \theta$ corresponding to $\omega_{i}$. Typically, we match sets of noise-corrupted feature obeervations (from another abstract space $\mathrm{Z}^{f}$ which forms the remainder of the matebing function domain) over time to elements in the firat apace. Unfortunately, these claseical decision theoretic functions may give higher. litelihoods than ideal for the wrong target clase, in part because kinematically unlikely aspect angles and aspect angle transitions over time are allowed.

The key to the proposed approach is to reatrict the domain of each function $\mathcal{C}_{i}$ further, requiring the target aspect angle over time to be consitent with the observed kinematics, since this restriction (correctly executed) should not advereely affect function velues for meanurements from the correct target class, but may lower the value for mearurements from incorruct target classes. Note that for any likelihood function corresponding to target class $w_{i}$, with the kinematically unrestricted and reatricted matching domains denoted respectively by $\mathbf{S}_{i}$ and $\mathbf{T}_{\boldsymbol{i}}$, we can thow by contradiction that:

for $\mathbf{T}_{\mathbf{i}} \subseteq \mathbf{S}_{\mathbf{i}}$ :

$\sup \mathcal{L}_{i}\left(\mathbf{T}_{i}, \mathbf{z}^{f}\right) \leq \sup \mathcal{L}_{i}\left(\mathbf{S}_{i}, \mathbf{z}^{f}\right)$

If the kinematic restriction is done properly, and the meanurements $\mathrm{Z}^{f} \subset \mathrm{Z}^{f}$ do arise from class $\omega_{i}$, the rentricted domain should etill include the region of highest origin likelihood, and the restricted likelihood function values should tend to be equal to the unrestricted function values. If the measurements come from some other clase $\omega_{j}$, however, the restricted likelihood function values are more likely to be reduced. Thus, restricted likelihood functions promise better recognition. As we will show, the Le Chevalier and Mieras approaches move in this direction by restricting the matching function do main to be consintent with feavible kinematics, or, in a cuboptimal fanhion consistent with observed kinematics. By further, optimal restriction using observed kinematics, we will achieve a more highly "tuned" likelihood funa tion (by analogy with a matched filter in the frequency domain). Restricting the matching domain of the likelihood function according to kinemation is the analog of conditioning $p\left(\omega_{i} \mid \mathbf{z}_{k}^{f}\right)$, were it known, on the added information given by kinematic measurements $Z_{m}^{L}$.

\section{Target Aspect Angle From Kinematics}

The relationship between aupect angle and kinematics is strong for many target clawes and has been exploited in sensor-augmented target tracken $[17,1]$. For conventional aircraft in particular, appet angle changes generally precede obnervable kinematic changes, as an aircraft rolls and creates an angle of attack for lateral acceleration to change its flight path. For this rouson, researchers usually seek to improve kinematic state catimates using aspect angle information from pose ertimates. In the sense of L\&P, bowever, we will use kinematic information to define the "a priori" probabilities $p\left(x_{0}^{k}\right)$ and $p\left(x_{k+1}^{k} \mid x_{k}^{0}\right)$ for transitions in an appect angle state space (hence the superecript $a$ on $x$ ), or in the DTW sense, to define nominal trajectories through that state space.

As implied in the previous paragraph, aircraft aspect angle estimates from standard kinematic trackers using prior and current measurements may well be in error by 90 or more degrees at the start of a maneuver. On the other hand, most conventional aircraft (and other target claness also), once committed to a turn, will generally hold that turn for eeveral seconds to gain the desired trajectory change. Thus, to determine the atate of an aircrafl at some time $t$, we can use kinematic information not only prior to $t$, but for eeveral meconds afterward as well. Since our intention is to gain all pousible information about the state of a turning aircraft, generally uaing information from a conventional extended Kalman filter (EKF) tracker, a natural step is to process the EKF outputs through an optimal fixed lag smoother (FLS) [23:16-17]. This wad the basic approsech in our research using outputs from a tracking rader simulation performed using the "Multimode Simulation for Optimal Filter Evaluation" (MSOFE) software [9], postproceseed by a FLS with a fixed lag of two to three seconds.

Fig. 1 shows EKF and FLS mean performance in eatimating one inertial component of target acceleration over 20 runs, where the true target acceleration in two $\mathrm{g}^{\prime} \mathrm{s}\left(64 \mathrm{ft} / \mathrm{sec}^{2}\right)$ during the period from three to eleven seconds and sero eleewhere. The upper solid curve is mean EKF error, while the lower solid eurve is mean FLS error. In practice, although the FLS did an excellent job of correcting EKF atate estimates as shown here, acceleration eatimates were still too noisy to provide amooth aspect angle estimates, particularly in atate directions where inoufficient true acceleration made use of the optimal amoother pointles [23:11]. Thus, second-order polynomials were fitted to the filter/moother poeition entimates, and differentiated twice to obtain an acceleration eatimate with error magnitudes that closely follow the mean FLS value in Fig. 1.

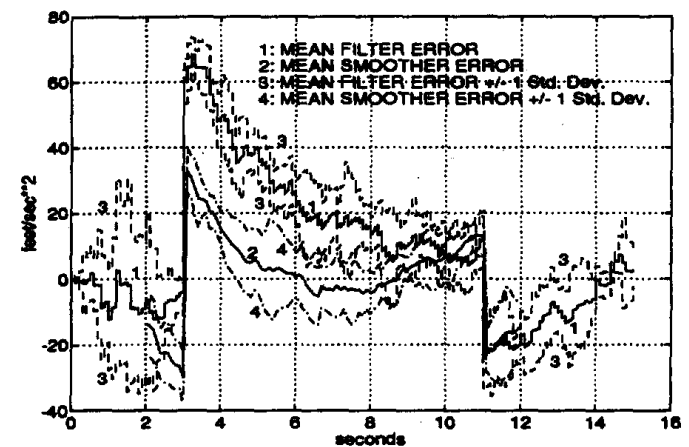

Figure 1: Kinematic State Eatimation Improvement by Smoothing

The dicadvantages of amoothing are added procesoing and the fact that our target information is no longer real-time. In general, we found that a high quality $(+/-20 \%)$ estimate of the target acceleration was obtained with a four-second delay - two weconds for the fixed lag amoother and two reconds for polynomial curve fitting. Following oneet of a major maneuver, 2-3 more seconde of delay are desirable to identify steady atate condition. (note how the FLS mean error curve in Fig. 1 begins to level out near the five second point). In any caec, so shown in Fig. 1, for a $2-\mathrm{g}$ turn lasting as little as eight ecconds, the target acceleration can be estimated with high confidence for approximately five seconds. An we will show below, the advantege accrued in better ponition and velocity state information can be well worth the wait and proceaning, particularly for turning accelerations in axcen of $1 \mathrm{~g}$.

Once the target velocity and acceleration atates are known and asumed to be in ateady atate relative to the target body frame, calculation of target- 
sensor aspect angle and aspect angle rate is straightforward for any set of assumptions on target control parameters. In our research to date, we have assumed a conventional aireraft's coordinated turn motion - for any control method in which the plane of the wings is essentially normal to the lift vector, minor deviations from the coordinated turn dynamics result only in an aspect angle position bias error which is ignored by our algorithms.

The kinematic state covariance estimate from the EKF/smoother allow: one to estimate the covariance of the kinematic aspect angle and aspect angle rate estimates, by use of the quadratic form:

$$
\mathbf{P}_{\mathbf{A}}=\mathbf{E} \mathbf{P E}^{T}
$$

where:

$P_{A}=$ is a $4 \times 4$ matrix, a first order (linearized) covariance estimate for the error in the angular position and angular rate of the nominal aspect angle, in the direction of and normal to the nominal aspect angle path.

$\mathbf{E}=$ is a 4 (row) $\times 6$ (column) Jacobian matrix of partial derivatives, defined by determining the partial derivative of angular position and rate along and normal to the nominal aspect angle path with respect to the target velocity and acceleration components along each inertial frame axis.

$P=$ is a $6 \times 6$ matrix, the filter/smoother-estimated covariance of the target inertial velocity and acceleration estimate

Straightforward extensions of this technique allow for calculation of angular state error "covariances" due to other variables. Due to unmodelled factors, the quantity in Eq. (9) can be treated as simply a lower bound on the true error covariance and a departure point for tuning

\section{The L\&P Approach and $p\left(\omega_{i} \mid Z_{k}^{f}, Z_{m}^{d}\right)$}

The purpose of this section is to apply Bayes' Rule [22], the L\&P methodology (see Sect. 3.2 and [18]) and aspect angle state transition information given by kinematic state estimates, to provide the a posteriori probability $p\left(w_{i} \mid Z_{k}^{f}, Z_{m}^{d}\right)$ for each class $\omega_{i}$, or a reasonable replacement.

Consider a set of $J$ a priori known target classes $\omega_{i}$, each represented by a target model having appropriate signature distributions associated with each aspect angle value. Given some discretization of continuous aspect angle on the targets (assumed the same for all target classes), each discrete aspect angle value is considered a state $\boldsymbol{x}^{a}$. Now, for any given problem (i.e., any given measurement set $\left\{\mathbf{Z}_{k}^{f}, \mathbf{Z}^{d}\right\}$ over some time interval), we can restrict our concern to a given aspect angle "windown or region - that is we assume a negligible probability that the class presented aspect anglea outside this region over the duration of the time interval corresponding to measurements $\mathbf{Z}^{f}$. Due to the smoothing process, the time interval corresponding to the kinematic measurements will generally contain the time interval of the signature measurements. The regions or windows may not be identical from class to class.

Now, define the super-region $\mathcal{X}^{a}$ as the superset of all aspect angle cells or states that belong to the region of consideration for at least one target class, a total of say $N$, cells or states in number. Any set of $k+1$ aspect angle cells, or aspect angle state history, corresponding for analysis purposes to discrete locations at signature measurement times along an aspect angle sequence which yields the $k$ signature measurements $\mathbf{Z}_{k}^{f}=\left\{\mathbf{s}_{1}^{f}, \mathbf{s}_{2}^{f}, \mathbf{z}_{3}^{f}, \ldots, \mathbf{s}_{k}^{f}\right\}$, wil be denoted $X_{k}^{a}=\left\{x_{0}^{a}, x_{1}^{a}, x_{2}^{a}, x_{3}^{a}, \ldots, x_{k}^{a}\right\}$ (where $x_{0}^{a}$ is an a priori or starting state and the other $k$ states correspond one-for-one to the signature measurements $\mathbf{Z}_{k}^{f}$ ).

The (finite) number $N_{p}$ of possible such aspect angle sequences through $X^{*}$ is given by the number of permutations of $N$ things taken $k+1$ at a time, with replacement, or $\left(N_{s}\right)^{k+1}$ sequences. We will denote the set of all such sequences as $\mathcal{X}_{p k}^{a}$. Henceforth, this development will refer to a particular "nth" sequence of $\boldsymbol{k}+1$ states as $\mathbf{X}_{k, n}^{a}$ (consistent with the notation $\omega_{i}$ referring to an $i$ th target class). Clearly, from the definition of $\mathcal{X}^{a}$, some of these sequences $X_{i, n}^{a}$ are of negligible probability for one or more target classes, because they fall outside the subsets of $\mathcal{X}^{a}$ appropriate for those classes. Other sequences are of negligible probability for all classes because they are kinematically unlikely.

We will see that applying the L\&.P equations (with reasonable modifications for target recognition) for any one model $\omega_{i}$ gives the particula state history $\mathrm{X}_{k, n}^{e}$, say $\hat{\mathrm{X}}_{k / w_{i}}^{L P}$ (i.e., the "L\&\&" estimate of the aspect angle sequence for model $\omega_{i}$ ), which maximises the conditional probability $p\left(\mathbf{X}_{k, m}^{a} \mid \mathbf{Z}_{k}^{f}, \mathbf{Z}_{m}^{d}, \omega_{i}\right)$. With appropriate modifications, we will be able to find the joint conditional probability $p\left(\mathbf{X}_{k, n}^{a}, \omega_{i} \mid \mathbf{Z}_{k}^{f}, \mathbf{Z}_{m}^{d}\right)$, which we will sum over all possible $\mathbf{X}_{k, n}^{a}$ to find the quantity that we desire, $p\left(\omega_{i} \mid \mathbf{Z}_{k}^{f}, \mathbf{Z}_{m}^{d}\right)$. The object here is to understand the relationship between (1) the information given by the L\&P approach, i.e., $\hat{\mathbf{X}}_{k / \omega_{i}}^{L P}$ for a particular $\omega_{i}$ and a joint conditional probability associated with that state sequence and (2) the information that we want, $p\left(\omega_{i} \mid \mathbf{Z}_{k}^{f}, \mathbf{Z}_{m}^{d}\right)$
Further assumptions are:

(1) Following L\&P, assume that $\Sigma_{j}^{f}$ is independent of $x_{i}^{a}$ and $x_{i}^{f}$ for $t_{j} \neq t_{l}$. This assumption is readily relaxed, at the risk of added computation. Each sf will ideally include measurements from independent sensors and feature spaces.

(2) It should be clear, and kept in mind during this development that, for any $l, p\left(\mathbf{Z}_{l}^{f} \mid \mathbf{Z}_{l-1}^{f}\right)=p\left(\boldsymbol{x}_{l}^{f} \mid \mathbf{Z}_{l-1}^{f}\right)$, and analogously that $p\left(\mathbf{X}_{l, n}^{a} \mid \mathbf{X}_{l-1, n}^{a}\right)=$ $p\left(x_{l, n}^{a} \mid \mathbf{X}_{l-1, n}^{a}\right)$.

(3) We assume that the "a priore" aspect angle state (cell) transition probability $p\left(x_{j+1, n}^{a} \mid x_{j, n}^{a}, Z_{m}^{d}, \omega_{i}\right)$ and probability of starting cell location $p\left(x_{0, n}^{a} \mid \mathbf{z}_{m}^{d}, \omega_{i}\right)$ are given by the EKF/smoother determination of mean aspect angle, aspect angle rate, and associated covariance estimates as discussed in Sect. 5 (i.e., from kinematic information). Alternate approaches are discussed in [20].

(4) We assume that $p\left(\omega_{i}\right)$ (a priori) is known for each target class $\omega_{i}$, and furthermore that $p\left(\omega_{i}\right)=p\left(\omega_{i} \mid Z_{m}^{d}\right)$, that is, that the kinematic measurements and derived kinematic state history provide no information as to the nature of the target. This last assumption is clearly neither true nor desirable if characteristic trajectories for various target types are classified probabilistically, and in that event another application of Bayes' Rule will incorporate this information into the ATR decision. In this development, however, we wish to assess recognition improvement due to DP sequence comparison methods only, so all targets are considered equally likely to have executed the observed maneuvers.

(5) For the high range resolution radar signature case, we assume that uncertainties in range bin alignment and scale factor uncertainty are handled by finding $\operatorname{MAX}\left\{p\left(z^{f} \mid x^{a}, \omega_{i}\right)\right\}$ for any combination of signature measurement $f$ and trial aspect angle state $x^{a}$ on any model $\omega_{i}$, essentially following the "maximum likelihood" method discussed in [33], and used in $[2,25]$

6.1 Relationship of $p\left(\mathbf{X}_{k, n}^{a} \mid \mathbf{Z}_{k}^{f}, \mathbf{Z}_{m}^{d}, \omega_{i}\right)$ to $p\left(\omega_{i} \mid \mathbf{Z}_{k}^{f}, \mathbf{Z}_{m}^{d}\right)$

Recall that Larson and Peschon sought the state history or sequence $\mathbf{X}_{k, n}^{a}$ to maximize $p\left(\mathbf{X}_{k, n}^{a} \mid \mathbf{Z}_{k}^{f}\right)$ in some general state space. Analogously in our case, trying to find a "best" state sequence in aspect angle space over some model $\omega_{i}$, we might seek a state history to maximize $p\left(\mathbf{X}_{k, n}^{a} \mid \mathbf{Z}_{k}^{f}, \mathbf{Z}_{m}^{d}, \omega_{i}\right)$. Following the L\&P approach with the above assumptions, we find (where the denominator term is given in the usual fashion by summing the numerator expression over all $\mathbf{X}_{k, n}^{a}$ ):

$$
\begin{aligned}
& p\left(\mathbf{X}_{k, n}^{a} \mid \mathbf{Z}_{k}^{f}, \mathbf{Z}_{m}^{d}, \omega_{i}\right)= \\
& \quad \underline{p\left(\mathbf{z}_{k}^{f} \mid \mathbf{X}_{k, n}^{a}, \omega_{i}\right) p\left(\mathbf{X}_{k, n}^{a} \mid \mathbf{X}_{k-1, n}^{a}, \mathbf{Z}_{m}^{d}, \omega_{i}\right) p\left(\mathbf{X}_{k-1, n}^{a} \mid \mathbf{Z}_{k-1}^{f}, \mathbf{Z}_{m}^{d}, \omega_{i}\right)}
\end{aligned}
$$

Thus for any given target model $\omega_{i}$, we can conceptually use the L\&P approach to find the set of states $\mathbf{X}_{k, n}^{a}$ which maximises $p\left(\mathbf{X}_{k, n}^{a} \mid \mathbf{Z}_{k}^{f}, \mathbf{Z}_{m}^{d}, \omega_{i}\right)$

So far, we have defined L\&P-like conditional probabilities for the aspect angle space corresponding to one target class $\omega_{i}$. Now, we consider the priori probability of class membership $p\left(\omega_{i}\right)$, and define the desired a posteriori probabilities $p\left(\omega_{i} \mid \mathbf{Z}_{k}^{f}, \mathbf{Z}_{m}^{d}\right)$. Assuming (with reservations as discussed above) that $p\left(\omega_{i}\right)=p\left(\omega_{i} \mid \mathbf{Z}_{m}^{d}\right)$, we start with the a priori probability $p\left(\omega_{i}\right)$ and then multiply by $p\left(x_{0, n}^{a} \mid \mathbf{Z}_{m}^{d}, w_{i}\right)$, and continue as in the L\&P development, to obtain in an analogous fashion (where the denominator is obtained by summing the numerator over all target classes and state sequences):

$$
\begin{aligned}
& p\left(\mathbf{X}_{k, n}^{a}, \omega_{i} \mid \mathbf{Z}_{k}^{f}, \mathbf{Z}_{m}^{d}\right)= \\
& \frac{p\left(\mathbf{z}_{k}^{f} \mid \mathbf{x}_{k, n}^{\mathrm{d}}, \omega_{i}\right) p\left(\mathbf{x}_{k, n}^{d} \mid \mathbf{X}_{k-1, n}^{d}, \mathbf{Z}_{m,}^{d}, \omega_{i}\right) p\left(\mathbf{X}_{k-1, n}^{a}, \omega_{i} \mid \mathbf{Z}_{k-1}^{f}, \mathbf{Z}_{m}^{d}\right)}{p\left(\mathbf{z}_{k}^{f} \mid \mathbf{Z}_{k-1}^{f}, \mathbf{Z}_{m}^{d}\right)}
\end{aligned}
$$

Now sum Eq. (11) over all possible $X_{k, z}^{a}$ for any given $\omega_{i}$ to obtain:

$$
p\left(\omega_{i} \mid \mathbf{Z}_{k}^{f}, \mathbf{Z}_{m}^{d}\right)=\sum_{\mathbf{X}_{\mathbf{i}, 1}}^{N_{p}} p\left(\mathbf{X}_{k, n}^{a}, \omega_{i} \mid \mathbf{Z}_{k}^{f}, \mathbf{Z}_{m}^{d}\right)
$$

Thus, the desired $p\left(\omega_{i} \mid \mathbf{z}_{k}^{f}, \mathbf{z}_{m}^{d}\right)$ can be found rigorously only by keeping track of, and performing appropriate calculations for, all possible aspect angle sequences over all possible target models- that is, all $\mathbf{X}_{k, n}^{a}$ in $\chi_{p k}^{a}$ over all $\omega_{i}$ (an exhaustive computation).

\subsection{Relationship of $I^{*}\left(\mathbf{x}_{k}^{a}, k\right)$ to $p\left(\omega_{i} \mid \mathbf{Z}_{k}^{f}, \mathbf{Z}_{m}^{d}\right)$}

Recall that the the L\&P method as implemented on different target models $\omega_{i}$ would find the $\mathbf{X}_{k, n}^{k}$, say $\hat{\mathbf{X}}_{k / \omega_{i}}^{L P}$, for each $\omega_{i}$, that maximises 
$p\left(\mathbf{X}_{i, a}^{a} \mid \mathbf{Z}_{k}^{f}, \mathbf{Z}_{m}^{d}, \omega_{i}\right)$, not by maximieing this conditional probability directly, but rather by maximising $I^{*}\left(x_{k}^{a}, k\right)$, where, in our form:

$$
\begin{aligned}
& \operatorname{MAX}_{x_{k, n}^{a}} I^{*}\left(x_{k, n}^{a}, k \mid w_{i}\right)= \\
& \underset{x_{k, n}^{a}}{\operatorname{MAX}}\left[p\left(z_{k}^{f} \mid x_{k, n}^{a}, \omega_{i}\right) p\left(x_{k, n}^{a} \mid x_{k-1, n}^{a}, z_{m}^{d}, \omega_{i}\right) I^{*}\left(x_{k-1, n}^{a}, k-1 \mid \omega_{i}\right)\right]
\end{aligned}
$$

Maximising this quantity rather than the conditional probability is desirable because we avoid having to compute values for all $X_{i, n}^{a} \in X_{p k}^{a}$, which we would have to do to find the denominator in Eq. (10). Examining $I^{*}\left(x_{k, n}^{a}, k \mid \omega_{i}\right)$ closely, note that the preceding equation is equivalent to:

$$
\underset{\mathbf{x}_{k, n}^{a}}{\operatorname{MAX}} I^{*}\left(\mathbf{x}_{k, n}^{a}, k \mid \omega_{i}\right)=\underset{\mathbf{X}_{k, n}^{a}}{\operatorname{MAX}}\left[p\left(\mathbf{X}_{k, n}^{a}, \mathbf{Z}_{k}^{f} \mid \mathbf{Z}_{m}^{d}, \omega_{i}\right)\right]
$$

Thus, the L\&P equations give the maximum $p\left(\mathbf{X}_{k, n}^{a}, \mathbf{Z}_{k}^{f} \mid \mathbf{Z}_{m}^{d}, \omega_{i}\right)$ and the state history entimate $\mathrm{X}_{k / \omega_{i}}^{L P}$ for a given $\omega_{i}$ which gives that maximum joint conditional probability density

\subsection{Approximating $p\left(\omega_{i} \mid Z_{k}^{f}, Z_{m}^{d}\right)$ (Likelihood Fcns.)}

Defining conditional probabilities for all tracks $\mathbf{X}_{k, n}^{a}$ over all target models $\omega_{i}$ is not practical. Modifying the right side of Eq. (12) to take summation over sets of $X_{k, n}^{a}$ 's rather than all (e.8., proper subsets of $X_{p k}^{a}$ ) for each $\omega_{i}$ in Eq. (12) creates a limiting process, so that as we converge toward summations over all $\mathbf{X}_{k, n}^{a} \in \mathcal{X}_{p k}^{a}$ for each $\omega_{i}$, the modified term converges toward the desired probability $p\left(\omega_{i} \mid \mathbf{Z}_{k}^{f}, \mathbf{Z}_{m}^{d}\right)$. We recognise that mont of the sequences will contribute little to the final probability - by definition, the sequence which contributes the most for each $w_{i}$ is the one given by the L\&P algorithm, $\mathbf{X}_{k / w_{i}}^{L P}$. Therefore, if we make the (extreme) choice of approximating the desired $p\left(w_{i} \mid z_{k}^{f}, Z_{m}^{d}\right)$ using only one sequence $X_{k, n}^{a}$ for each $w_{i}$, the most reasonable wch approximation would be given by (note the "hat" over $p$, denoting an estimate).

$$
\hat{p}\left(\omega_{i} \mid \mathbf{z}_{k}^{f}, \mathbf{z}_{m}^{d}\right)=\frac{p\left(\hat{\mathbf{X}}_{h \omega_{\omega_{i}}}^{L L P}, \mathbf{z}_{k}^{f}, \omega_{i} \mid \mathbf{z}_{m}^{d}\right)}{\sum_{\omega_{2}}^{J} p\left(\hat{\mathbf{X}}_{k / \omega_{j}}^{L P}, \mathbf{z}_{k}^{f}, \omega_{j} \mid \mathbf{Z}_{m}^{d}\right)}
$$

where, using the appropriate $I^{*}\left(x_{k, n}^{a}, k \mid \omega_{i}\right)$ for each $\omega_{i}$;

$$
p\left(\hat{\mathbf{X}}_{k / w_{i}}^{L P}, \mathbf{Z}_{k}^{f}, \omega_{i} \mid \mathbf{Z}_{m}^{d}\right)=\left[\underset{\operatorname{MAX}_{k, n}^{a}}{\operatorname{Mat}} I^{*}\left(\mathbf{x}_{k, n}^{a}, k \mid \omega_{i}\right)\right] p\left(\omega_{i}\right)
$$

Progressively better approximations to $p\left(\omega_{i} \mid z_{k}^{f}, z_{m}^{d}\right)$ would be given by adding the contributions from other aspect angle sequences for each $w_{i}$ : for example, those that pass through points $\mathbf{X}_{k, n}^{a}$ in progressively larger neighborhoods around the points in $\mathbf{X}_{k}^{L} / \omega_{i}$ for each $\omega_{i}$.

At this point, the Le Chevalier and Mieras algorithms evidently can be described as "suboptimal" application of this L\&P approach. Lacking explicit information on a priori aspect angle state transition probability $p\left(x_{j+1, n}^{a} \mid x_{j, n}^{a}, z_{m,}^{d}, w_{i}\right)$ and probability of starting cell location $p\left(x_{0, n}^{a} \mid Z_{m}^{d}, \omega_{i}\right)$ given by a kinematic tracker/moother (or other source), they effectively replace the former quantity with a uniform probability denity, or "fixed bound" on allowable associations, the extent of which is defined by aspect angle transition bounds allowable by vehicle kinematics (the Mieras bounds are biased in the direction expected from obwerved kinematics). The latter quantity $p\left(x_{0}^{0}, \mid Z_{m}^{d}, w_{i}\right)$ is treated as equal for all starting states, or effectively ignored.

As our result will wow, this suboptimal approach can improve recognition significantly by restricting the wild aspect angle transitione (and unreasonable low cont matches) attempted on incorrect target claseses by an independent-look (conventional matching algorithm) recogniser working in a noiay rignature domain. However, these "anpect angle bound" algorithmo can allow apparent appect angle transitions that are inconaistent with the observed kinematics, such as aspect angle sequences that atop or move in the opposite direction to that consistent with observed motion.

These effects are often exhibited in our tents when incorrect matches are attempted, and suggeat that aubsequent processing of these aspect angle equences is warranted to improve classification. The Mieras algorithm is believed to apply such a form of proceasing [24], which may be a significant improvement over the approach of Le Chevalier et al. [19]. It in important to note that the teats conducted by Le Chevalier et al. wrere conducted in a one-dimensional (great circle) aspect angle space, where this "wandering" would have been less noticeable.
The apparent advantage accrued by these "espeet angle bound" approaches is that, theoretically, they can be made to be "real-time", since they do not require as long a time delay as that required to develop the kinematic aspect angle rate estimate, or $p\left(x_{j+1, n}^{n} \mid x_{j, n}^{n}, z_{m,}^{d}, \omega_{i}\right)$. As we noted above, however, for aircraft targets, body angular rates can be on the order of hundreds of degrees per second, and are unobservable to the kinematic trackere generally used to find "global" aspect limits for any recognition algorithm. Thus, it seems clear that one may need to accept delays of up to a few seconds and some form of smoothing to provide any reliable aspect angle eatimates. If the target is determined to be turning during this period, that kinematic information can and ahould be used explicitly.

Recalling our comments in Sect. 3.3, it is also clear that the potential shortcoming of a L\&P-type approach as applied to target recognition (e.g. Eq. (15), making decisions based on but one set of $k$ aspect angle states per target model) is that, asauming equal a priori probubilities for each target class $\omega_{i}$, the sequence of states which yields the highest $I^{*}\left(x_{k, n}^{k}, k \mid w_{i}\right)$ over all $\omega_{i}$ may not fall on the particular target class $\omega_{i}$ which has the highest (true) $p\left(\omega_{i} \mid \mathbf{Z}_{k}^{f}, \mathbf{Z}_{m}^{d}\right)$. It may be that one model bas a particular aet of aspect angle atates with associated signatures, such that the "best" sequence $\dot{X}_{k / w_{i}}^{L P}$ traverses these points and given this class the highest $p\left(\mathbf{X}_{k_{n}, n}^{\mathbf{a}}, \mathbf{Z}_{k}^{f} \mid \mathbf{Z}_{m}^{d}, \omega_{i}\right)$, but if all possible $\mathbf{X}_{k, n}^{e}$ are considered, this class is less likely to have been the origin of the observed signatures $\mathbf{z}_{k}^{f}$ than some other. Hence the desire as implied above, to investigate the contribution from multiple paths.

An alternate approach, then, is to construct sets of trajectories through the state apace over the time frame of interest, uning the same information on aspect angle from kinematics used to provide $p\left(x_{j+1, n}^{a} \mid x_{j, n}^{a}, Z_{m}^{d}, \omega_{i}\right)$, the a priori information for L\&P-type approaches. These trajectories then imply sequences of feature observations, which can be compared to the observed sequences using DTW techniques. Further, in a departure from usual DTW, we can allow the "best peth" to move from one trajectory to another. This defines "two-dimensional" form of DTW.

\section{Implementing Motion Fusion}

Our research to date has involved seven basic algorithms, five of which use forms of DP sequence comparison for motion fusion, and two of which are expected to provide upper and lower bound on performance. For the high range resolution radar (HRRR) domain in which this concept was evaluated, we used a Mahalanobis distance metric, which treats the ERRR measurement or "aweep" as a vector of Gauasian elements - this is by far the most popular HRRR metric to date $[15,25,26,32]$.

Consistent with the Mahalanobis metric approach, HRRR signatures were treated as 128-element (range "bin") vectors, downsampled from higher dimenuioned vectors (choosing maximum return of " $n$ " elements) output by the signature generator [8] in $\mathrm{dBam}$ for desired aspect angles, polarisations center frequencies, and bandwidths. Independent Gaussian noise realisations of constant variance were then added to each bin, using statistics gathered by analysis of actual HRRR tests [4] with a modified vervion of an eximting program [26]. The amumption of noive independence from bin to bin was simply for convenience, rince our test data showed significant crose-bin correlation (standard deviation was retuonably constant at 5-7 $\mathrm{dBsm}$ along range and apect angle extente). Since a maximum likelihood approach to range regiatration and constant noise variance were used, the signature comparison proceas was effectively a weighted correlation. In passing, we must note that the statintics of BRRR aignatures deserve further research.

\subsection{Independent Look (IL) Algorithm}

This is a conventional decision theoretic target recogniser as discussed in Sect. 2. No restrietion is placed on the "pose estimate" so generated - we find MAX $\ln \left\{p\left(m_{k}^{f}\left\{x_{k, n}^{*}, \omega_{i}\right)\right\}\right.$ within the apecified appect angle window at each measurement time $t_{k}$.

\subsection{Perfect Knowledge of Aspect (PKA) Algorithm}

This algorithm providen an upper bound on recognition performance in that it asoumes that the recogmiver knows perfectly the brue aspect angle over time for each target class executing the obeerved maneuver. For the Mahalanobis metric, this figure is simply the $\log _{\text {e }}$ joint maximum (classical) likelihood for known or true aspect angle $x_{i, k}^{k}$ over time, $\ln \left\{\prod_{k=1}^{K}\left[p\left(s_{k}^{f} \mid x_{i, k}^{k}, \omega_{i}\right)\right\}\right\}$.

\subsection{Fixed Bound (FB) Algorithm}

This algorithm is an implementation of the Le Chevalier algorithm, as that approach is believed to work: the algorithm has no information from kine- 
matics on the expected direction of aspect angle change, but knows that the change is bounded. No subsequent processing is applied.

\subsection{Full Larson and Peschon (L\&P) Algorithm}

This algorithm finds the natural log of the term in Eq. (14). Note that the numerical values of this algorithm include contributions due to the a prior aspect angle transition probabilities $p\left(x_{j+1, n}^{a} \mid x_{j, n}^{a}, z_{m}^{d}, w_{i}\right)$. Contributions associated with the L\&P "a priori" state $x_{0, n}^{a}$ were not included, to provide for unbiased comparison of the curves.

\subsection{One- and Two-Dimensional Path Warping}

The one-dimensional path warping algorithm defines continuous onedimensional paths in aspect angle, parallel to and including the "nominal" path given by the EKF/amoother kinematic estimate. One-dimensional, unrestricted endpoint $[27,28]$ DTW is performed along each trajectory. The basic form requires continuous matching (no deletions). Each local path cost is normalised by the total number of associations along that path This is a departure from usual DTW practice, and can lead to violations of the "Principle of Optimality" [6], but worked well in our tests, since for the proper match of measurements to target class, and constant measurement noise statistics across the target length, the local average matching cost is expected to be near the global average (see discussion in [20])

Two-dimensional path warping uses the same set of trajectories defined for the one-dimensional case, but local continuity constraints allow the optimum path to move from one trajectory to another. Other factors are as for the one-dimensional case.

\section{Performance Evaluation Approach}

All of the algorithms discussed thus far are in fact interpretable in terms of likelihood functions as defined by Rao [29]. Following the development in [23:96-101], we now introduce the use of generalised ambiguity functions for assessing maximum likelihood estimator performance, as developed by Schweppe [31:376-381] and Maybeck [21]

\subsection{The Generalized Ambiguity Function}

The generalised ambiguity function (GAF) is defined by the equation:

$$
\mathcal{A}_{k}\left(\boldsymbol{\Omega}, \boldsymbol{\Omega}_{\mathbf{t}}\right) \equiv \int_{-\infty}^{\infty} \cdots \int_{-\infty}^{\infty} L\left[\boldsymbol{\Omega}, \boldsymbol{Z}_{k}\right] f_{\mathbf{Z}\left(\boldsymbol{t}_{k}\right) \mid \boldsymbol{\Omega}\left(\boldsymbol{t}_{k}\right)}\left(\boldsymbol{Z}_{k} \mid \boldsymbol{\Omega}_{\mathbf{t}}\right) d \boldsymbol{Z}_{k}
$$

where:

$\mathbf{n}_{\mathbf{t}}=$ the particular combination of states $\mathbf{x}(t)$ and parameters $\mathbf{y}$ (the latter generally constant over the time interval of interest) for the truth system which generates the set of all possible measurement histories $\boldsymbol{Z}_{k}$ over which the integral is taken. A likelihood function $L$ defined for $\boldsymbol{n}_{\mathbf{t}}$, operating on an element of this measurement history set, will ideally generate a higher value than will any $L$ defined for some other value of $\boldsymbol{\Omega}$, operating on an element of this measurement history set (the ambiguity function evaluates the extent to which this is true in the mean)

$\mathbf{n}=$ state/parameter values for which the likelihood function is defined, for evaluation against measurements generated by a truth model with state and parameter values $\boldsymbol{n}_{\mathbf{t}}$

$\mathcal{A}_{\mathbf{k}}\left(\boldsymbol{\Omega}, \boldsymbol{\Omega}_{\mathbf{t}}\right)=$ the generalised ambiguity function, a function of $\boldsymbol{\Omega}$ for $\mathrm{a}$ given $\boldsymbol{n}_{\mathbf{t}}$ and likelihood function $L$

$\boldsymbol{L}\left[\boldsymbol{\Omega}, \boldsymbol{Z}_{\boldsymbol{k}}\right]=$ the likelihood function, a function of $\boldsymbol{Z}_{\boldsymbol{k}}$ when defined for a given $\boldsymbol{\Omega}$ (note that the script " $Z$," or $\boldsymbol{Z}$, is used as the "dummy" form of $\mathbf{Z}$, appropriate for showing functional relationships in an integrand)

$f_{Z(t)} \mid \mathbf{n}\left(t_{k}\right)\left(Z_{k} \mid \boldsymbol{n}_{t}\right)=$ the probability density function of the measurements, given that the true states and parameters have the value $\boldsymbol{n}_{\mathbf{t}}$

$\mathcal{Z}_{k}=$ the measurement history vector as of time $t_{k}$

Thus, the GAF is the expected value of likelihood functions defined for combinations of states and parameters, conditioned on the true states and parameters having particular values. For any particular value of $\boldsymbol{\Omega}$ defining the litelihood function, there is in fact a distribution of likelihood function values produced, due to the different realizations of measurements produced by a syatem with true states and parameters $\boldsymbol{\Omega}_{\mathbf{t}}$. Ambiguity functions can be developed analytically for some likelihood functions $[21,23]$, or in any case empirically by experiment or Monte Carlo simulation (as in our case).

Examining the ambiguity function for each realisable value of $\boldsymbol{\Omega}_{t}$ and for each such value of $\boldsymbol{\Omega}_{t}$, a range of $\boldsymbol{\Omega}$ encompassing reasonable state and parameter values expected other than at $\boldsymbol{\Omega}_{t}$, we desire that the function have an easily discernible global maximum at $\boldsymbol{\Omega}_{t}-$ i.e, that local maxima, if present, are "widely" separated from the global maximum at $\boldsymbol{n}$.
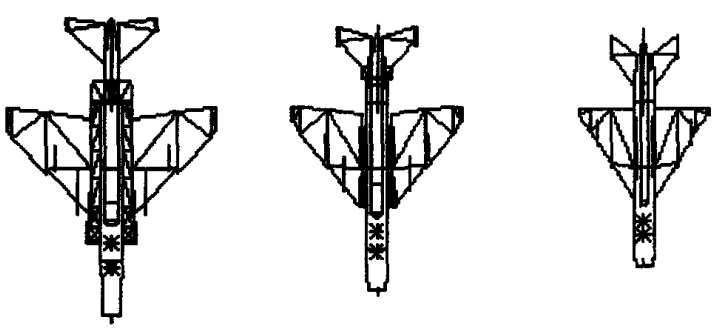

Figure 2: F-4, 50\% Morph, and MIG-21

The curvature of the GAF at $\mathbf{\Omega}$ can be related to the Cramér-Rao lower bound (CRLB) [29] of the covariance for a state/parameter estimate obtained by the use of that likelihood function $[23,31]$. Recent interest has been directed toward Cramér-Rao-like lower bounds for multisensor fusionbased target tracking [10], but evidently no analogous bound has been defined for multisensor target recognition [7].

\subsection{Applying the Generalized Ambiguity Function}

In general, to obtain the GAF in a Monte Carlo fashion, we define one likelihood function for each point of interest in state/parameter space. Each ikelihood function then operates on measurements from a system at some "true" state/parameter point, unknown to the likelihood functions a priori. The mean values of the likelihood function over a large number of measurement sets define the GAF. In classical ATR, the state (kinematic, temperature, etc.) of the candidate targets is readily defined, but real targets define only discrete points in some infinite-dimensional, generally continuous "parameter" space defined by their physical shape, materials, etc. Use of the GAF in target recognition, then, requires the definition of pseudo-targets in some sense "in-between" real targets of interest.

To develop these pseudo-targets, we first defined parent targets, or "points" in the (abstract) parameter space used by our target signature generator [8]. Each parent was defined by the same number of shapes and surfaces, but the locations occupied in 3-D space by these objects differed according to the size and shape of the respective target. Borrowing from the language of computer graphics [5], then, 3-D linear interpolation "morphs" (morphological, or shape, transformations) were performed to obtain new "points" in target parameter space, or new targets in some sense "between" the two pareats. Fig. 2 shows an F-4 Phantom II and a MIG-21 as parent targets, and a peeudo-target defined by $50 \%$ interpolation between the parents. It must be emphasized that this linear interpolation was never expected to translate into linear changes of the likelihood function outputs, and it did not.

\section{Results and Discussion}

Parent target classes like those in Fig. 2 are readily separable in the feature opace and metric used here with any of the algorithms shown. More ambiguous scenarios which demonstrate the power of the proposed approach were generated by defining similar parents, high noise, and small morph fractions. Typical outputs obtained in this way are shown in Figs. 3 and 4, for which the parent target classes were a MIG-21 (the true target) and an SU-22 augmented with scatterers. The dotted vertical lines in the first figure indicate parameter (target) interpolation values for which likelihood functions were defined, and spline curve fits connect the generalized ambiguity function (GAF) values to provide the curves shown. The second figure shows percent correct recognition performance for likelibood functions tuned for morph (parameter) values other than the true (unknown target) value (a correct recognition is taken as one for which the properly tuned function outputs a higher likelihood than the improperly tuned function). Relevant target trajectory parameters are shown.

Note that the Independent Look (IL) algorithm defines the lower bound on performance (worst), and the Perfect Knowledge of Aspect (PKA) algorithm defines the upper bound (best). The Fixed Bound (FB) algorithms provide significantly improved separation from the IL result, but the algorithms which fuse filter/smoother-provided kinematic information generally provide equal or better separation in each case. Performance of the 1-D and 2-D Warp algorithms is somewhat degraded in this simulation because these methods force contiguous matches for measurements taken artificially 


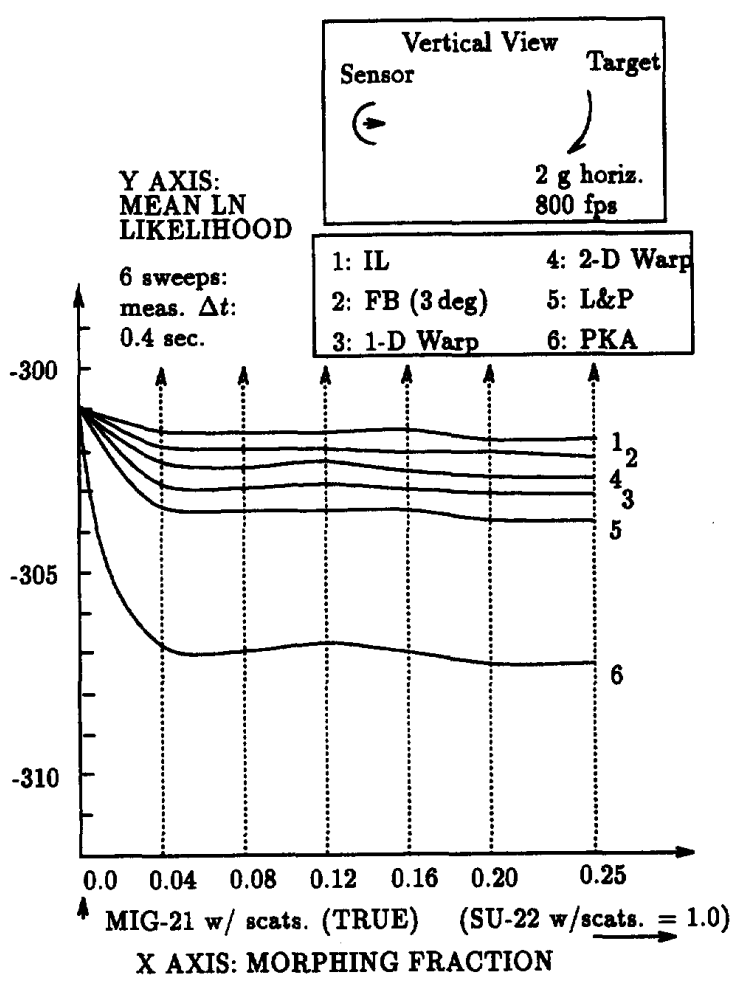

Figure 3: Generalised Ambiguity Function

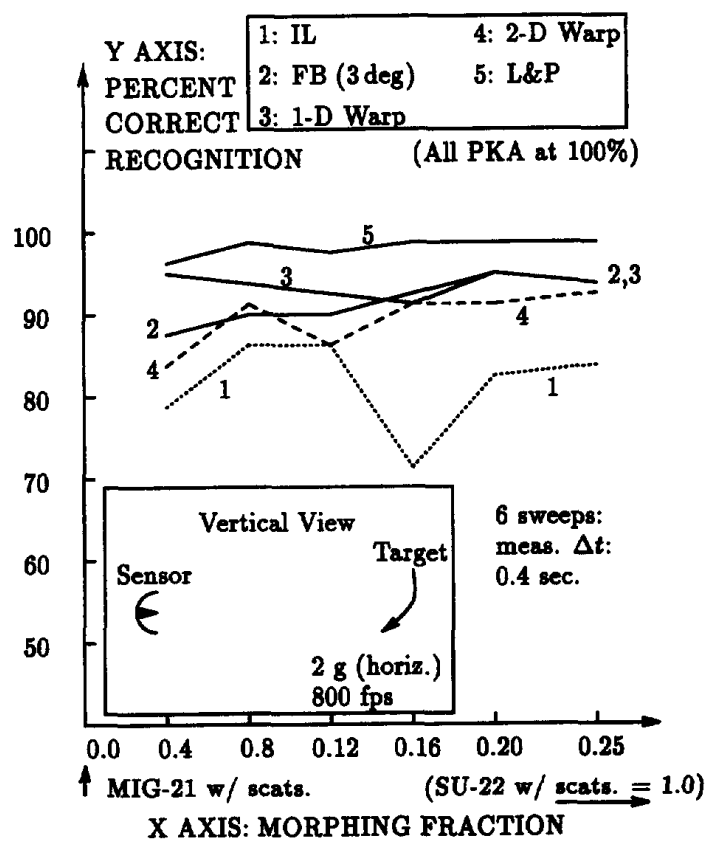

Figure 4: Percent Correct Recognition

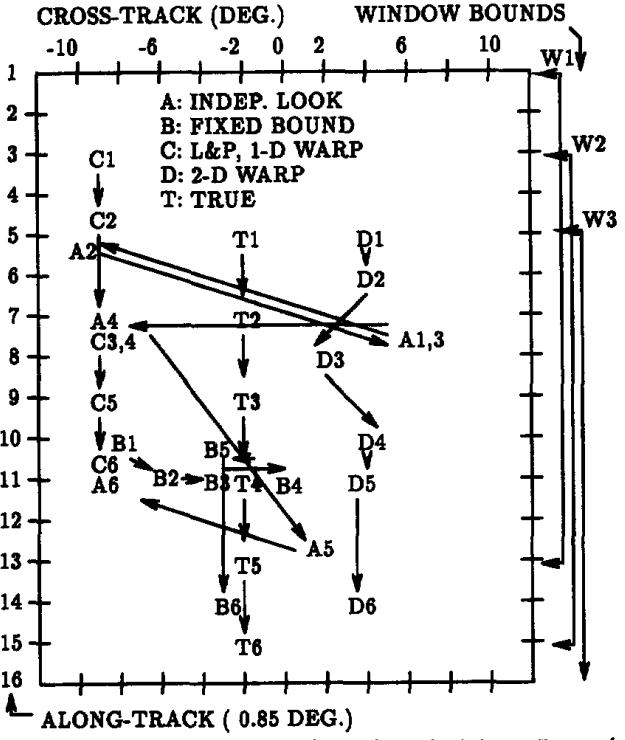

Figure 5: ML Aspect Angle Estimates (Paths) on the Wrong Target (numbers show sequence order for six sweeps)

from particular discrete aspect anglea. Results and anomalies are discussed in detail in [20].

Fig. 5 shows why progressive domain restriction provides better separation when measurements from one target class are matched to the library for another (i.e., wrong) class. This figure represents a region of solid angle in target aspect defined by the union of six "windowe" or aspect angle bounda for any one meanurement. The ML aspect angle locations identified by several algorithms over this angular extent for six measurements are shown the true aspect angle is shown as well. Note the erratic appect sequence selected by the IL processor, and the still rather unlikely sequence selected by the FB algorithm. The DTW and L\&P-based algorithms select more likely (linear) aspect angle paths, but their predilection to follow kinematicallyreasonable pathe forces a higher matching cost (lawer likelihood) for this incorrect model-to-target association. In contrast, for measurements matched to their true origin target class, the different algorithms were much more likely to aseociate with the same aspect angle region.

The improvement from kinematic information fusion increases with the mean aspect angle rate or $g$ level of the target's turm. As turn rate increasea, physics limits the number of possible aspect angle states $x^{*}$ (and therefore state aequences $X_{a}^{a}$ ), and we can limit the remaining matching domain even more sevorely to (fewer) equuences of expected length and direction. For the FB algorithm (with a fixed anmpling rate), however, we must open the aspect angle bounds to give it any chance of tracking the nominal aspect rate on the true target. This increases dimensionality and gives it a greater chance of finding an improperly-high likelihood match on an incorrect target model. Other approaches for identifying infeasible aspect angle sequences may mitigate this problem, but may not effectively use the information available in observed kinematics.

Converaely, as turn rate decreaces, the small mean aupect angle rate available to motion fusion algorithms tends to produce the same results as the FB algorithm, which asoume no mean rate, and can use small bounds when a amall mean rate exits. For a sero-mean turn rate estimate, FB algorithms provide an effective approach - this is simply the limiting case of the L\&EP algorithm for a sero-mean, uniform $p\left(x_{j+1, n}^{a} \mid x_{j, m}^{q}, Z_{m,}^{d}, w_{i}\right)$. For an aspect angle rate known to be sero, conventional decision theoretic recognition for a fixed apect angle is most effective - this is in turn the limiting case of the FB algorithm for a bound of sero degrees.

Changes in the parameter space due to the morphing process can create apparently anomalouw results, e.8., cases where the measurements from an F-4 were clower in Mahalanobis metric sense to sweeps from the MIG than they were to aweepe yielded by an interpolated target only $25 \%$ removed from the P-4. These cases resulted from the relative motion of scatterer: during the morphing procesa, and were found to be physically reasonable after investigation. Modified morphing rules can resolve these anomalies.

Likelihood function differences for targets of interest (i.e., points of inter- 
est in parameter space) are the key design criterion, but quick rolloff around the design point of each likelihood function should be of high secondary interest. The advantage to evaluating ML target recognition systems with GAFs is clearly that the method allows us to evaluate the curvature of the likelihood function away from its design point. This rolloff is directly related to the Cramér-Rao lower bound for the estimator used to develop the GAF [23]: practical evaluation of this bound using our approach requires one to generate target "morphs" or interpolations arbitrarily close to the design point $\Omega_{t}$, and evaluate the behavior of the GAF in this region.

The limiting value of the CRLB for these estimators is evidently given by the CRLB found in this fashion for the PKA algorithm (i.e., joint maximum likelihood for known aspect angle over time). In any case, the figures indicate that the separability of any two target classes may depend on factors other than behavior of the GAF near the true target parameter point. Therefore, this CRLB concept is perhaps not of greatest interest where we simply wish to identify a set of measurements as belonging to one of several a priori known points in some parameter space. The CRLB may be most useful where we wish to perform classical parameter estimation: for example, quantifying the extent to which we can estimate the optimum location in some finite-dimensional, model-based target parameter space to represent a previously unclassified real target, known only by measurements.

\section{Further Directions and Conclusion}

The dynamic programming-based approaches discussed here for exploiting the joint likelihood of kinematic and nonkinematic information in object recognition are just part of a class of techniques discussed more fully in [20]. Generically, these techniques are Bayesian multiple model parameter estimators [23:129-136] using linear and nonlinear models that exploit the unique coupling between states and parameters for different classes, an extension both of (1) the efforts of a previous student of the second author [17] and (2) independent observations by Daum [3:177-178] made subsequent to the definition of this research.

The research described here has illuminated significant new directions for research in multisensor fusion and target recognition. Multisensor fusion of target kinematic and signature information is an exceptionally promising field. This fusion process can be viewed as exploiting the syntax of physical processes, the joint likelihood of observable events, or restricting the domain of likelihood functions - in any case, it is clear that proper implementations of such fusion must provide recognition performance equal to or better than that of conventional "independent look" techniques.

\section{References}

[1] Andrisani, Dominick, et al. "A Nonlinear Helicopter Tracker Using Attitude Measurements," IEEE Trans. Aerospace and Electronic Systems, 27(1):40-47 (January 1991).

[2] Bar-Shalom, Yaakov, editor. Multitarget-Multisensor Tracking: Advanced Applications. Norwood, MA: Artech House, 1990.

[3] Bar-Shalom, Yaakov, editor. Multitarget-Multisensor Tracking: Advanced Applications, II. Norwood, MA: Artech House, 1992.

[4] Beckner et al., F. Automatic Radar Target Identification (ARTI) Phase II (U). Technical Report WRDC-TR-90-1003, Wright-Patterson AFB, OH: General Dynamics (Pomona) and U.S. Air Force Wright Labs, March 1990.

[5] Beier, Thaddeus and Shawn Neely. "Feature-Based Image Metamorphosis," Computer Graphics, 26(2):35-42 (July 1992).

[6] Bellman, Richard E. and Stuart E. Dreyfus. Applied Dynamic Programming. Princeton, NJ: Princeton University Press, 1962

[7] Bhanu, Bir and Terry L. Jones. "Image Understanding Research For Automatic Target Recognition." Proceedings: Image Understanding Workshop. 249-254. Defense Advanced Research Projects Agency (DARPA), January 1992.

[8] Bramley, M., et al. User's Guide to RCSTooLLBox Version 2.0. Technical Report 168, Massachusetts Institute of Technology Lincoln Laboratory, September 1991. Lincoln Manual 168.

[9] Carison, Neal A., et al Pragram Design Description for a Multimode Simulation for Optimal Filter Evaluation (MSOFE). Technical Report AFWAL-TR-88-1137, Avionics Laboratory, Air Force Wright Aeronautics Laboratory, February 1989.
[10] Daum, Frederick E. "Fundamental Limits in Multisensor Data Fusion." Proceedings of the 1990 IEEE International Conference on Systems Engineering. 316-319. 1990.

[11] Dougherty, Edward R. and Charles R. Giardina. Mathematical Methods for Artificial Intelligence and Autonomous Systems. Englewood Cliffs, NJ: Prentice Hall, Inc., 1988.

[12] Dreyfus, Stewart E. and Averill M. Law. The Art and Theory of Dynamic Programming. New York: Academic Press, Inc., 1977.

[13] Ford, Roger. "State of the Art... Steering Tracked Vehicles," Armed Forces, 4(5):195-196 (May 1985).

[14] Fu, King-Sun. Syntactic Pattern Recognition and Applications. Englewood Cliffs, N.J.: Prentice-Hall, Inc., 1982.

[15] Fukunaga, Keinosuke. Introduction to Statistical Pattern Recognition (Second Edition). Boston, MA: Academic Press, 1990.

[16] Gorman, John W., et al "Partial Shape Recognition Using Dynamic Programming," IEEE Trans. Patt. Analy. and Mach. Intel., 10(2):257266 (March 1988).

[17] Kendrick, Jerry D., P.S. Maybeck, and J.G. Reid. "Estimation of Aircraft Target Motion Using Orientation Measurements," IEEE Trans. AES, 17(2):254-259 (March 1981).

[18] Larson, R.E. and J. Peschon. “A Dynamic Programming Approach to Trajectory Estimation," IEEE Trans. Automatic Control, 11(3):537540 (July 1966).

[19] Le Chevalier, François, et al. "Radar Target and Aspect Angle Identification." Proceedings of the IEEE 1978 International Conference on Pattern Recognition. 398-400. 1978.

[20] Libby, Edmund W. Application of Sequence Comparison Methods to Multisensor Data Fusion and Target Recognition. $\mathrm{PhD}$ dissertation, U.S. Air Force Inst. of Tech., 1993.

[21] Maybeck, Peter S. Combined State and Parameter Estimation for OnLine Applications. $\mathrm{PhD}$ dissertation, Massachusetts Institute of Technology, 1972.

[22] Maybeck, Peter S. Stochastic Models, Estimation, and Control: Volume 1. New York: Academic Press, 1979.

[23] Maybeck, Peter S. Stochastic Models, Estimation, and Control: Volume 2. New York: Academic Press, 1982.

[24] Mieras, Harry. "Conversations with the Author Regarding Raytheon Efforts on Dynamic Programming for Integration of High Range Resolution Radar Data." (discussed article in 1990 CISC Proceedings), December 1991

[25] Mieras, Harry and Timothy Cichocki et al. "Airframe Identification Results Using High Range Resolution." 1990 Joint Service Combat Identification System Conference Technical Proceedings (U), edited by Dean Youngberg (Chairman). 1107-1115. 1990. (SECRET/NOFORN).

[26] Mitchell, Rick et al. "Conversations with the Author Regarding High Range Resolution Radar Target Recognition." (input from Wright Laboratory Target Recognition Technology Branch), 1991.

[27] Parsons, Thomas W. Voice and Speech Processing. New York: McGraw-Hill, Inc., 1987.

[28] Rabiner, L.R., et al. "Considerations in Dynamic Time Warping Algorithms for Discrete Word Recognition, ${ }^{n}$ IEEE Trans. ASSP, $26(6): 575$ 582 (December 1978).

[29] Rao, C.R. Linear Statistical Inference and Its Applications. New York: John Wiley and Sons, Inc., 1973.

[30] Sankoff, David and Joseph B. Kruskal. Time Warps, String Edits, and Macromolecules: the Theory and Practice of Sequence Comparison. Reading, Massachusetts: Addison-Wesley Publishing Co., Inc., 1983.

[31] Schweppe, F.C. Uncertain Dynamic Systems. Englewood Cliffs, NJ: Prentice Hall, 1973.

[32] Stewart, Clayton, et al "Comparison of classification approaches for high-range resolution radar." Automatic Object Recognition II, Proc. SPIE 1700. 146-155. 1992

[33] Weiss, Anthony J. and Benjamin Friedlander. "Efficient Dynamic Programming in the Presence of Nuisance Parameters," IEEE Trans. AES, 25(2):277-280 (March 1989). 